\title{
Bibliographie von Aspergillus. \\ 1729 bis 1928.
}

\author{
von \\ Hiroshi Tamiya und Shinkichi Morita.
}

(FORTSETZUNG XVII.)

\section{Autorenregister I.}

Die Ziffern stellen die laufenden Nummern der Arbeiten und die Jahre (in Klammern), in denen die betreffenden Arbeiten erschienen, dar.

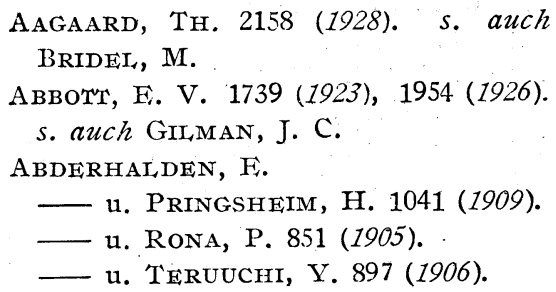

AAGAARD, TH. 2158 (1928). s. auch BRIDEI, M.

Аввотт, E. V. 1739 (1923), 1954 (1926).

s. auch GILmaN, J. C.

ABDERHALDEN, F.

— u. Pringsheim, H. 1041 (1909).

— u. RoNA, P. 851 (1905).

u. TERUUCHI, Y. 897 (1906).

ABEL, R. U. BUTTENBERG, P. 602 (1899). AB'T, G. 2036 (1927), 2159 (1928).

ADAMETZ, L. 279 (1886).

ADAMS, J. F. s. MANNS, T. F.

ADERHOLD, R. 603 (1899).

AFANASSJEWA, M. S. KOSTYTSCHEW, S. AGULHON, H. 1094 (1910).

— u. SAZERAC, R. 1204 (1912).

Ahi,bURG u. Matsubara, S. 184 (1878).

Akagi, T., Nakajima, I. u. T'sugane,

K. 1451 (1915).

Akama's

AIBERTINI $u$. SCHWEINITZ. 2294. (1805).

ALBERTOTTI, G. 989 (1908).

AIEXANDRESCU, N. S. SION, V.

ALGER, 794 (1904).

AMBRož, A. 1142 (1911).

AMElung, H. 2637 (1927).

AMmoN, R. s. RONA, P.

Amons, W. J. Th. 1522 (1917), 1656 (1921).

ANDERSEN, C. W. 2038 (1927).

ANDERson, C. 1834 (1925).

ANDERSON E. G. F. S. BUNKER, J. W. B. ANDO, F. 1295 (1913).

ANIRES. 2160 (1928).

ANGERER, K. v. u. HAR'TMANN, A. 1954
(1926).

AokI, K. $s$. Dot,, $\mathrm{H}$.

APPENDIX, M. 1885 (1925).

ArCHANGELSKY. 2161 (1928).

ARChIBALD, R. G. 1296 (1913).

ARcichowskrJ, V. 942 (1907).

ARMSTEAD, D. u. HARLAND, S. C. 1740 (1923).

ARMSTRONG, G. M. 1657 (1921). s. auch DUgGaR, B. M.

ArNaudi, C. 2162 (1928).

ARNOLD, I, s. JORI,ING, J. W.

ARTAULT, S. 406 (1893).

AsarnoJ, S. s. EULER, H. V.

Aso, K. 604 (1899). 637 (1900).

ATkinson, R.W. 213, 214 (1881), 227(1882).

AURAND. $s$. ROLLET, FTIENNE.

Avetita, C. S. Baccarini, P.

A. W. 852 (1905).

AXENFELD, TH, $S$. UHTHOFF, W.

Ayers, S. H. $s$. THOM, CH.

AzUMA, T. 1896 (1925).

BACCARINI, P. 1143 (1911).

- u. AveTTA, C. 263 (1885).

BACH, A. s. ChodaT, $\mathrm{R}$.

BACH, D. 1802, 1803, 1804 (1924), 2163 (1928).

BACHMANN, F. M. S. WALKER, J. C.

BAHR. 1485 (1916).

BAIL. S. NEES V. ESENBECK, C. G.

BAIL, TH. 107 (1867).

BAIrEY, F. M. 1043 (1909). 
BAIII, P. s. SARTORY, A.

BAINIER, G. 202, 203 (1880), 215 (1881), 943 (1907), 990 (1908).

- u. SARTORY, A. 204, 205 (1880), 991 (1908), 1043 (1909). 1144, 1145, 1146 (1911), 1205, 1206 (1912), 1297 (1913).

s. auch SARTORY, A.

BAKER, J. C. S. SHERMAN, H. C.

BAKES, W. E. $s$. ThaYSen, A. C.

BaldwiN, M. E. s. Sherman, H. C.

BALI. 2336 (1901).

BALLAND u. DRoz. 1044 (1909).

BANCROFT, K. 2366 (1913).

BANDMANN, S. 432 (1894)

BARNES, B. 2164 (1928). s. auch GWYNNEVAUGHAN, H. C. I.

BARNUM, C. C. S. LEE, H. A.

BARTETZKO, H. 1095 (1910).

BARY, A. DE. 66 (1854), 94 (1866), 116, 117 (1868), 127 (1870), 216 (1881), 228 (1882), 256 (1884), 293 (1887).

u. WORONIN, M. 95 (1866), 128 (1870).

BAUMGARTEN, P. 327 (1889).

- u. MÜLLER, R. 229 (1882).

B̈̈UMLER, J. 294 (1887).

BAY, I. S. MOREL, A.

BAZIN. 1523 (1917).

BeaUregard, H. 565, 566, 567 (1898).

BEAUVERIE, J. 660 (1901), 740 (1903). s. auch Hollande, A. $\mathrm{CH}_{\mathrm{H}}$.

BECQUEREL, P. 1096 (1910).

BEDFORD, H. W. 1741 (1923)

BEHDJEL, H. $S$. HODARA, M.

BEHRENS, J. 385 (1892), 447 (1895), 487, 488 (1896), 741 (1903).

BEILLE, I. 1207 (1912).

BELIN, P. 1956 (1926).

BELI UGGI, L. 2412 (1925).

BENDIXEN, H. C. 2165 (1928).

BENECKE, W. 433 (1894), 448 (1895), 489 (1896).

Bennet, C. W. S. Young, H. C.

BERBERICH, F. M. $s$. BURR, A.

BERKELEY, M. J. 34, 35 (1836), 38 (1838), 67 (1855), 74 (1857), 83 (1860), 136 (1873), 145 (1874), 153 (1875).

- u. BROOME. 54 (1851), 154 (1875). - 1. CURTrs, M. A. 61 (1853), 121 (1869). s. auch Cooke, M. C.

BERLESE, A. N. 328 (1889).
BERLINER, C. 2166 (1928).

BeRNHAUER, K. 1805 (1924), 1957, 1958, 1959 (1926), 2167, 2168, 2169, 2170, 2171 (1928).

— u. SchöN, K. 2172 (1928;

u. Wolf, H. 2173 (1928).

BERSCH, W. 1097 (1910).

BERTHELOT, A. 407 (1893), 944 (1907).

Bertrand, G. 1<08, 1209, 1210 (1912), 1387 (1914).

- u. HoldERER, M. 104.5 (1909), 1098. 1099 (1910).

- u. JAVILLIER, M. 1147, 1148, 1149 (1911), 1211, 1212 (1912).

-, Rosenblatit u. Me RosenBLATT. 213, 1214 (1912), 1298 (1913).

Bertrand, J. s. Gosse't, A.

BESSON, A. 528 (1897).

BEST. 898 (1906).

BESTA, C. 853, 854 (1905).

s. auch CENI, C.

BEXMA THOE KINGMA, F. H. VAN. 2174 (1928). s. auch FAI,CK, R.

BEZOLD, F. 137 (1873).

BEZSSONOF, N. 1551 (1918), 1577 (1919), 1616, 2383 (1920).

Bidot, CH. s. LiAvier, G.

BIEN, Z. S. STORM VAN IEEUWEN, W. Bierry, H. u. Coupin, F. 1299 (1913).

BiJL, P. A. VAN DER. 1517 (1920), 1658 (1921).

BischofF, G. W. 84 (1860).

BLANC, G, u. CAILLON, I. 1806 (1924). BlANCHARD, R. 365 (1891), 490 (1896).

BLOCHWI'TZ; A. 1300 (1913), 1388, 1389 (1914), 1742 (1923), 1887 (1925), 2175, 2176 (1928).

Br,OME, W. H. 899 (1906).

BLUMENTRITT, F. 661 (1901), 855 (1905). BLÜMMEL, F. S. FREUDENBERG, $\mathrm{K}$.

BoAs, F. 1452 (1915), 1486 (1916), 1524, 1525 (1917), 1552, 1553 (1918), 1578, 1.579, 1580, 1581 (1919), 1696 (1922), 2039(1927).

— u. LEBERLE, H. 1554 (1918), 1582 (1919).

— u. MERKENSCHLAGER, F. 1743 (1923).

BoDIN, E.

— u. GaU'TIER, L. 900 (1906).

- u. LENORMAND, C. 1215 (1912).

BOEDIJIN, K. B. 2177 (1928). 
BÖESEKEN, J. u. WATERMAN, H. I. 1216 (1912).

BöKE, J. 122 (1869).

BOKORNY, TH. 1453 (1915), 1487 (1916), 1526 (1917), 1583, 1584 (1919), 1697(1922).

Bolognesi, G. u. Chiurco, A. G. 1744 (1923).

BOMMER, E. u. ROUSSEAU, M. 192(1879). BONDORFF, K. A. 1555 (1918).

BONJEAN, E. 742 (1903).

BONNET, R.

—, Duquénois, P. u. Vincent, G.

1960 (1926). s. auch TERROINE, É. F.

BONNIER, G. 1150, 1151 (1911).

BONORDEN, H. F. 86 (1861).

BONORDEN, M. 55 (1851).

BORCHERT, A. 1951 (1026).

BORdAS, F. 796 (1904).

BORNAND, M. 1301 (1913).

Borsani, E. P. s. CASTrilion, B. A.

BORTELS, H. 2040 (1927).

s. auch RIPPEL, A.

Bory u. DURIEU DE MAISONNEUVE. 2297 (1846).

Borzi, A. 242 (1883).

BOSELLI, J. 1152 (1911).

Boston, I. N. 662 (1901).

BÖTTGER, H. 1659 (1921).

BOUdIER, E. 96 (1866).

BOUFFARD. 856 (1905).

BOULANGER-DAUSSE, F. 529 (1897).

BOURQUHLOT, É. 243 (1883), 408, 409, $410,411,412,413$ (1893), 434 (1894), $449,450,(1895), 491,492,493$ (1896), 568 (1898).

- u. BRtDEL, M. 1660 (1921).

u. HÉRISSEY, H. 451, 452, 45.3 (1895), 494 (1896), 569 (1898), 857.(1905).

Bovo, P. 901 (1906).

BRANDEN, F. VAN DEN, u. MOREELS, W. 2041 (1927).

BranNon, J. M. 1745 (1922).

Brault, A. u. LOeper, M. 7.97 (1904). BRAULT, J. 992 (1908).

BREFELD, O. 134, 135 (1872), 146 (1874), 155(1875), 164(1876), 217(1881), 993(1908).

u. TAVEL, F. v. 366 (1891).

BREMER, W. 691 (1902). s. auch KÖNIG, J. u. SPIECKERMANN, A.

BRENNER, W. 1153 (1911), 1390 (1914), 1454 (1915).
BRESADOLA, A. J. 743 (1903).

Bresciani, G. 1807 (1924).

BRICK, C. 1302 (1913).

BRIDEL, M.

- u. AAGAARD, TH. 2042, 2043(1927). s. auch BOURQUELOT, É.

BrIGHT, T. B., Morris, L. E. u. SUMMERS, F. 1808 (1924).

BRIZI, U. 945 (1907), 994 (1908).

BROCQ-RousSEU, D. 995 (1908). s. auch STOYKowiTsCH, M.

Brooks, C. 902 (1906). - , Fisher, D. F. u. CoOLEY, J. S. 1391 (1914).

BROOME. S. BERKELEY, M. J.

Brown, W. M. A. 1698 (1922).

BR'TNIK, A. 1488 (1916).

BRUMPT, E. 903 (1906).

BRUNSTEIN, A. 663 (1901).

BRUNTZ u. SARTORY. 1392 (1917).

Bruschi, D. s. Pan'taneli, E.

BuBÁk, F. 664 (1901). s. auch KaBÁt.

Buchanan, R. E. u. FUtMeR, E. I. 2178 (1928).

BUCHNFR, E. 386 (1892).

BUCKLEX, J. S. s. MOHLER, J. R. u. Church, M. B.

BUI,

BUlitaRd. 5 (1780), 6 (1791).

Bunker, J.W.B. u. ANDERSON, E.G.E. 2179 (1928).

Bunting, R. H. 1809 (1924), 2044 (1927).

Bur. 996 (1908).

BUrgess, P. S s. LipMaN, CH. B.

Burgess, R. 1810 (1924), 2180 (1928).

BURNETT, H.R. S. REVIS, C.

BurNs, A. C. 2045 (1927).

BURNSIDE, C. 2181 (1928).

BURoMskiJ, I. 1217, 2362 (1928).

BUrr, A., WolfF, A. u. Berberich, F. M. 1219 (1912).

BÜSGEN, M. 264 (1885).

BUTJagin, P. W. 798 (1904).

BUTKEWITSCH, W. 744 (1903), 1047 (1909), 1699, 1700, 1701, 1702 (1922), 1746, 1747 (1923), 1811, 1812 (1924), 1888, 1889 (1925), 1962 (1926), 2046 (1927).

- u. OrLow, W. G. 1703 (1922).

BUTLER, E. J. 1556 (1918).

BUTTENBERG, P. S. ABEL, R.

BXALL, S. s. KOPELOFF, N. 
Cacace, E. 665 (1901).

CAILION, L. S. BLANC, G.

Calderon, L. 367 (1891).

CaldWewL, M. I. s. Sherman, H. C.

CAlMETTE. 2317 (1892).

CAMP, A. F. 1748 (1923).

CAmurri, I. V. 1100 (1910).

Camus, L. 530 (1897).

CAO, G. 570 (1898).

Carapelite, E. 997 (1908).

Carbone, D. 1219 (1912).

- u. MARINCOLA-CATTANEO, R. 998 (1908).

u. Rusconi, M. 1101 (1910).

Carnevale-Ricci, F. 2047 (1927).

Carnót, P., Gérard, P. u. Rathery, F. 1618 (1920).

CARo, I,UIGI DE. 2182 (1928).

CASE, E. M. u. MCCullagh, D. R. 2183 (1928).

CASelia, A. S. Nuvole'Tti, G.

CastagNe. 56 (1851).

Casteliani, A. 1220 (1912), 1303 (1913), 1749 (1923), 1890 (1925).

Castrillon, E. A. u. Borsani, E. P. 1891 (1925).

Catoire, M. s. Malfítano, G.

Catsaras, J. 1221 (1912).

Cattaneo, A. 193 (1879), 244 (1883), 2318 (1892).

u. Oliva. 2313 (1888).

Cavara, V. 2184 (1928).

Cazejusis. s. Galavieline.

Ćelakovsky, L. F. 1222 (1912).

CENI, C. 745, 746, 747, 748 (1903), 799 (1904), 858, 859 (1905), 904 (1906), 946 (1907).

— u. BESTA, C. 692 (1902), 749 (1903), 800 (1904), 860 (1905).

Cerza, U. s. Grazia, S. DE.

Chable, R. 1527 (1917).

ChaLIENGER, F.

-, KLein, L., Surramaniam, V. u. WALKER, T. K. 2185 (1928).

-, Surramaniam, V. u. WaI,ker, T. K. 2048, 2049 (1927).

s. auch WALKER. T. K.

Chameers, H. S. s. Fraser, H. C. J. Chantemesse. 348 (1890), 368 (1891).

Chapman, A. C. 1661 (1921).

Chapman, $\mathrm{CH}_{\text {. }}$ u. Eitheridge, W. C.
2367 (1913).

Charpentier. 1355 (1915).

Charpentier, P. G. 861 (1905).

Chatelitier, L. s. NANTa, A.

Chevaliter, F. F. 24 (1826).

Chevaliter, P. S. EMILe-Weil, P.

Chrn, S. s. Nakazawa, R.

Chrurco, G. A. 1813, 1814, 2400 (1924). s. auch Bol,OGNESI, G.

Chodat, F. 1963, 1964 (1925).

Chodat, R. u. BaCh. A. 693 (1902).

Chrétiten. 1154, 1155 (1911).

Christmas. 414 (1893).

Chrzaszcz, T. 694 (1902).

Chudrakow, N. 495 (1896).

Chupp, Ch. 1892 (1925).

Church, M. B. 1750 (1923).

- u. BUCHLEY, J. S. 1751 (1923).

s. auch MAy, O. E., Oshima, K. u. THOM, CH.

Ciampi, N, 2186 (1928).

CiferRI, R. 1662 (1921), 1704 (1922), 2050, 2051 (1927).

Clark, A. B. s. RaIsTrick, H.

CLARK, F. M. 2052 (1927).

ClaARK, J. F. 605, 606 (1899), 695 (1902).

Clark, W. M. u. LubS, H. A. 1528(1917).

Cleland, J. B. 1815 (1924).

CLÉMENT, H. 1304, 1305 (1913).

ClEMENTS, F. E. 1048 (1909).

Clerc. S. SARTORY, A.

Clinton, G. P. 862 (1905).

Cocardas, E. 257 (1884), 265 (1885).

Codur. 863 (1905).

Codur, J. u. Thiry, G. 1305 (1913).

CoHen, C. 1619 (1920).

s. auch NEUBERG, C.

Corn, F. 258(1884), 315 (1888), 349(1890), 415, 415 (1893).

Cohnherm. 89 (1865).

Colas, A. 1049 (1909).

Colin, H. 2384 (1920).

— u. ESTIENNE, V. 1816 (1924.

Colirins, I. D. 2053 (1927).

Coliodi, A. M. 1102 (1910).

Committee Report Councir, on PharMACY. AND CHEMIS'TRY. 1223 (1912).

Compton, A. 1456, 1457 (1915), 1663(1921).

CoNDELII, S. 801 (1904), 1393 (1914),

1458 (1915), 1664 (1921).

Conn, H. W. 802 (1904). 
Cook, S. F. 1965, 1966 (1926).

Cooke, M. C. 90 (1865), 131 (1871), 156 (1875), 194 (1879), 206 (1880), 245 (1883), 266 (1885). 329 (1889).

U. BERKELEY, M. J. 147 (1874).

CoOley, J. S. S. Brooks, C.

COPPOCK, PH. D.

- , SUbramaniam, V.u. Walker, T.

K. 2187 (1928). s. auch WALKER, T.K.

CoRda, A. C. I. 36 (1837), 40 (1842),

Corpaci, A, 1893 (1925).

Costantin. 316 (1888).

- u. LUCET. 750 (1903), 864 (1905).

CotTetTe Fils A. u. BoIdin, A. 531 (1897).

Coupin, F. 1394 (1914).

s. auch BIERRY, H.

Coupin, H. 751, 752, 753, 754 (1903), 803 804 (1904), 1050 (1909), 1103 (1910), 1307 (1913), 2054 (1927).

- u. FRIEDEL, J. 805 (1904).

s. auch MolLIARD, M.

CoukT, D. 1395 (1914).

Cramer, C. F. 80 (1859), 97 (1862).

Criveiri, E. 1752 (1923).

C.rookshank, F. G. 2188 (1928).

Cruess, W. V. 1224 (1912).

Cugini, G. 165 (1876).

CURrey, Fr. s. WelWiTsch, F.

Currie, J. N. 1459, 1460 (1915), 1489 (1916), 1529 (1917). s. auch Тном, CH.

Curson, H. H. 1655 (1921).

CURTIS, M. A. $S$. BERKELEY, M. J.

Cusumano; A. 1894 (1925).

CZAPEK, F. 2334 (1899), 666 (1901), 696, 697 (1902), 999. (1908).

s. auch KoHN, E.

C.ziser, S. S. LINDNER, P.

DAISH, A. G. S. DAvis, W. A.

DALE, E. 1051 (1909), 1225 (1912), 1396 (1914).

DAMM, O. 1461 (1915).

DANGEARD, 'P. A. 805 (1907), 947 (1907), 1490 (1916).

DARESTE, C. 230 (1882).

DAUB, G. $s$. WII,SON, J. A.

Davis, A. R. s. Duggar, B. M.

Davis, W. A., DaIsh, A. G. u. SAWYer, G. C. 1491 (1916).
Davison, W. C. s. Masiow, H, I. u. WAKSMAN, S. A.

DEAN, A. I. 755 (1903).

DECL,ERCK, J. 1395 (1925).

DEGEN, A. 865 (1905).

DEILE, R. 807 (1904).

DELACROIX, G. 369 (1891), 418 (1893), 532 (1897), 905 (1905).

s. auch PATOUILIARD.

DELAS, R. s. LASSALLE, H.

DELBRÜCK, M. 2335 (1899).

Deleano, N. 948 (1907), 1000 (1908), 1052 (1909).

DERSChaU, M. 698 (1902).

DESLONGCHAMP, E. 39 (1841).

Desmazières, J. 32 (1834).

D'HERELLE, E., 2399 (1923). — (u. SMiTh, G. H.) 2401 (1924).

Diakonow, N, W. 280 (1886), 435 (1894).

Drxon, D. 2189 (1928).

DodGe, C. W. s. GÄUmanN, E.

DOEBELT, H. 1053 (1909).

DOEPNER, H. s. FRIEDBERGER, E.

Dol, H. u. AOKI, K. 1226 (1912).

DomaRADSkY, M. 1001 (1908).

Donal, D, R. u. SMITH, F. F. 1705 (1922).

Dox, A. W. 1054 (1909), 1104, 1105, 1106 (1910), 1156 (1911), 1227 (1912), 1397 (1914), 1462 (1915).

- u. GOLDEN, R. 1157 (1911).

u. MAYNARD, L. 1228 (1912).

- u. NEIDIG, R. E. 1158 (1911), 1229 (1912), 1308 (1913), 1398 (1917).

u. RoARK, G. W. 1620 (1920).

DREYFUSS, I. 436 (1894).

DROZ. S. BALLAND.

Dubois, R. 350 (1890).

DUbourG, F. s. GAyon, M.

DURREUIL, M. W. 2343 (1903).

DUCLAUX, M. E. 2309 (1883), 267 (1885), 330 (1889), 397 (1892), 571 (1898).

DUDE, M. 756 (1903).

D'UDEKEM. $S$. GLUGE.

DUGGAR, B. M. 667 (19.01), 1530 (1917). u. ARMSTRONG, J. K. 1896 (1925). u. DAvis, A. R. 1492 (1916).

DUQuénors, P. s. BONNET, R. U. TERROINE, É. F.

DURAND. $s$. WILDEMAN, E. DE.

Durieu, M. u. Mon'tagne, C. 47(1849). 
Dusch, T. v. u. Pagenstecher, A. 75 (1857).

E. u. E. 2327 (1896)

FCKLES, C. H., Fitch, C. P. u. SEAL, J. L. 1817 (1924).

EFFRONT, J. 388 (1892), 2392 (1922), 1818 (1924).

EHRENBERG, C. G. 2295 (1818).

EHRLICH, F. 906 (1906), 2368 (1913), 1531 (1917).

— u. JACOBSEN, K. A. 1159 (1911).

— u. LAANGE, F. 1399 (1914).

Fichelpaum. 295 (1887), 317 (1888), 949 (1907).

EIDAM, F. 231 (1882), 246, 247, 248 (1883).

EIGNER, A. $s$. KLEIN, G.

EINTHOVEN; W. s. STORM VAN LEEJWEN, W.

EKMAN, G. 1150 (1911).

EIFFING, FR. 351 (1890', 1557 (1918).

EIIASBERG, P. s. KosTytscheW, S.

EILIS, J. B. u. EVENHART. 281 (1886).

ËMILE-WEIL, P.

- u. Chevaliter, P. 205 ; (1927).

-, Chevalitier, P. u. Flandrin, P. 2056 (1927).

- u. GaUdin, I. 158.5 (1919).

—, Grégoire, R., Cheval,iter, P. u. FlandRIN P. 2419 (1927) GRÉGOIRE, R. u. FLANDRIN, P. 2057, 2419 (1927).

s. auch NANTA, A.

FMMERLING, O. 699, 700 (1902), 757 (1903).

FNGELKE, C. 701 (1902), 866 (1905).

EPIER, R. $s$. I,ESZCZYŃSKI, R. V.

Firickson, M. J. s. WAHL, E. F.

ERRERA, L. 607 (1899).

ESCHENHAGEN, F. 331 (1889).

ESSER, A. 1897 (1925).

ESTIENNE, V. 1819 (1924: s. auch Colin, H.

ESTOR, W. 2058 (1927).

ETheiridge, W. C. s. Chapman, $\mathrm{CH}$. EULER, H. V.

- u. AsarnoJ, S. 1586 (1919).

-, JOSEPHSON, K. u. SÖDERLING, B. 1820 (1924).

— u. KULLBERG, S. 1161 (1911).

- u. STAEHELIN, M. 1899,1900(1925).
EVENHART. s. EIIIIS, J. B.

FABER, F. C. von. 1055 (1909).

FABRE, G. 1162 (1911).

FALCK, R. 2402 (1924).

u. BFYMA THOE KINGMA, VAN. 1821 (1924).

- u. KAPUR, S. N. 1822 (1924).

F. A. M. 1753 (1923).

FARLOW, W. G. 185 (1878).

- u. Seymour. 318 (1888).

Faure, G. s. Pantaneiri, E.

Favat,oro, G, 2059 (1927).

Fawceit', H. S. s. Savastana, G,

F. C. 2304 (1876).

Ferton, I- D. u KaUfFManN, G. 2060 (1927).

FerdinandSen, C. C. F. 1107 (1910).

FERMI, C. 496 (1896), 572 (1898), 668 (1901). 950 (1907), 1056 (1909), 1309(1913). - u. Montesano, G. 454 (1895).

FERNBACH, A. 332 (1889), 352, 353(1890), 608 (1899), 638 (1900), 702 (1902).

Ferraris, T. 2371 (1914). - u. MASSA, C. 1230 (1912).

FERRY, R. 758 (1903).

FEURICH, G. 703. (1902).

FÉvre, E. $s$. ThOM, СH.

Fick, B. 2385 (1920).

-FIElirz, H. 1967 (1926).

Filosofov, M. S. u. Malinovski, V.E. 2190 (1928).

FindLAY, W. P. K, 2191 (1928).

Firel, I, V. s. Mou't, A.

FischER, A. 389 (1892), 2330 (1897). s. auch WINTER, G.

Fischer, B. 437 (1894).

Fischer, E. 249 (1883), 2192 (1928).

- u. ZEMPLEN, G. 2357 (1910).

FISH, S. 1968 (1926).

Fisher, D. F. s. Brooks, C.

FI'TCH, C. P. S. ECKI,ES, C. H.

Fi'TCH, R. 907 (1906).

Flament, L. s. Sartory, A.

Fl, ANDRIN, P. $s$. ÉMILE-WEIL, P.

FLIEG, O. 1706 (1922).

FLORENCE, G. $s$. HugouneNo, L.

FL,̈̈GGE, C. 497 (1896).

FOCKE, W. O. 1163 (1911).

For, J. G. $s$. SöHNGEN, N. L. 
FORNET, A. S. HERTER, W'.

FosSE, R. 1310 (1913), 1400 (1914).

Frank, A. B. $s$. LEUNIS, J.

Frank, TH. s. FREUDENBERG, K.

FrankE, H. 1967 (1926).

FrankLand, G. C. s. FRANKLAND, P. F.

Frankland, P. F. u. FRankiand, G. C. 2322 (1894).

Fraser, H. C. J. u. Chameers, H. S. C51 (1907).

Fred, E. B. S. Peterson, W. H.

FREEMAN, E. M. 2347 (1905).

FRESENIUS, G. 51 (1850), 62 (1853), 83 (1863).

FREUDENBERG, K. 2376, 2377 (1919), 2386 (1920).

-, BlüMMEL, F. u. Frank, TH. 2061 (1927).

- u. VOLLBRECHT, E. 2393 (1922).

- u. WALPUSKI, H. 2391 (1921).

FREY, A. 1970 (1926), 2062 (1927).

FREYD, A. 2193 (1928).

FRIEDBERGER, E.

U. DOEPNER, H. 952 (1907).

- u. GolDSCHMID, E. 1164 (1911).

FRIEDEL, J. 803 (1904), 867 (1905)。

s. auch Coupin, H.

FRIEDRICHS, O. v. 1463 (1915).

FRIES, E. M. 17 (1821), 27 (1828).

FrokHLICH, H. 1002 (1908).

Fromm, J. 1901 (1925).

Frouin, A. 1231 (1912), 1754 (1923).

- u. MERCIER, V. 1401 (1914).

FUCHS, E. 438 (1894).

FUCHS, J. 1971, 1972 (1926).

FUCKEL, L. 91 (1865), 123 (1869).

FUHRMANN, F. 1311 (1913).

FujiTa, E. s. Kurono, K.

FUlmer, F. I. s. BUChanAN, R. E..

FULTON, H. R. 908 (1906).

FUMAGALII, A. 953 (1907).

FUNKE, G. L. 1707, 1708 (1922), 1973 (1926), 2063 (1927).

GABBE, E. 2064 (1927).

Galavielie u. Cazejust. 1709 (1922)

GaleotTt, C. u. I.EVI, M. 1108 (1910).

GaLlEMAERTS, V. 1165 (1911).

GaLli-VAI,ERIO, B. 1057 (1909), 1109,
1110 (1910), 1755 (1923).

- u. Rochaz-DE JoNGH, J. 868 (1905), 909 (1906).

s. auch RABOW, S.

GararD, I. D. u. Sherman, H. C. 1558 (1918).

GARDEY, F. 17.56 (1923).

GARNIER, C. 759 (1903).

Gasparrini, G. 296, 297 (1887).

GAUDIN, L. S. ÉMILE-WEIL, P.

GaUdUchfau, A. 1111 (1910).

GäUMANN, E. 1974 (1926).

— u. DODGE, C. W. 2194 (1928).

GAUTIER, A. 148 (1874).

GAUTTER, L. 954 (1907).

s. auch BoDIN. E.

GAYON, M. 176 (1877), 186, 2305 (1878). - u. Dubourg, F. 282, 283 (1886). GEE, U. P. U. MASSEY, A. B. 1402 (1914). GEERLIGS-PRINSEN, H. C. 2325 (1897), 498 (1896).

GELONESI, G. 2065 (1927).

GENTNER. 1403 (1914).

Gerard P. $s$. Carnot, P.

GERBER, C. 533, 534 (1897).

Gibson, R. J. H. 354 (1890).

Giglioli, J. 232 (1882).

GILBERT, F. A. 2195 (1928).

GIIKINET, A. 157 (1875).

GILLOT, H. 609 (1899).

GIr,man, J. C. u. Аввот', E. V. 2066 (1927).

Giroud, P. S. Pasteur ValieryRADOT.

Glaser, H. u. Prinz, F. 1975 (1926).

GLOGER, R. 910 (1906).

GLUGE U. D'UDEKEM. 78 (1858).

GODDARD, H. N. 1232 (1912), 1312 (1913).

GOLDEN, K. E. 610 (1899).

GOLDEN, R. S. DOX, A. W.

Gor,DSCHMID, E. S. FRIEDBERGER, E. GOODWIN, T, C. u. HANGER, I. C. 1976 (1926).

Gosro, B. 390, 391 (1892), 911 (1905), 1058 (1909).

Gosi.INGS, N. 809 (1904).

Gosset, A., Ber'trand, J. u. MAGRoU, J. 2196 (1928).

Gössı. 810 (1904)

Go'oH, K. 1902 (1925).

GRAF, F. 639 (1900). 
Grafe, V. u. MAGistris, W. 1903(1925).

GRAMENIZKY, M. J 1112 (1910), 1313, 1314 (1913).

GRAWITZ, P. 177 (1877).

Grazia, S. S. DE u. CerrzA, U. 912(1906), 955, 956 (1907).

GREEN. 118 (1868).

GREGGIO, E. 1494 (1916)

Grégotre, R. S. ÉMILE-WEIL, P.

GREVIILE, R. K. 21 (1823).

GREZES. 1233 (1912).

GRIESEBACH, A. U. REINKE. J. 13\%(1873).

GRIJNS, G. 760 (1903).

GrimM, M. 1404 (1914).

GrohmanN, W. 259 (1887).

GRölLER, L. V. s. Kossowicz, A.

GRÖNI,UND; CHR. 195 (1879).

Grove, W. B. 268 (1885), 1532 (1917).

GRUNDHERR,.G. E. V. s. KUHN, R.

GRÜTER, W. 1405 (1914).

GUBERLET, J. E. 1757 (1923).

GUDDEN, B. 63 (1853).

GUÉGUEN, F. 573 (1898), 611 (1899), 811 (1907), 869 (1905), 1059, 1060, 2353(1909), 1113 (1910).

GULLLEMARD, A. 1315 (1913).

GUILLIERMOND, A. 761 (1903).

GUITTONNEAU, G. 2067 (1927).

GUSTAFson, F. G. 1587 (1919), 1621(1920).

GWYNNE-VAUGHAN, I.C.I. U. BARNES B. 2058 (1927).

HAENICKe, A. 1495 (1916).

HAENSEL ER, C. M. 1665 (1921).

HAGEM, O. 937 (1907).

HahN, A., LaVes, W. 1 . SchäHER, L. 1977 (1926).

HALBERTSMA, F. H. 319 (1888).

HALLAR, D. A. 2 (1742), 4 (1768).

HALLIER, E. 92 (1865), 97, 98, 99, 100, 101, 102, 103 (1866), 108 (1867). 119(1868), 124 (1869). 129 (1870).

HANGER, I. C. $s$. Goodwin, T, C.

HaNSEN, E. C. 166 (1876), 196 (1879), 284 (1886), 355 (1890), 455, 456 (1895), 535 (1897).

HaNSeN, K. 2197 (1928).

Hanus, J, u. Stockx́, A. 640 (1900).

HaNZAWA, J. 1166 (1911), 1234 (1912). - u. Miyauchi. 1167 (1911).
Hario', P. 392 (1892), 419 (1993).

HaRkNeSS, H. W. 269 (1885).

Harland, S. C. $s$. ARMSTead, D.

HARMER, D. 1588 (1919).

HARRISON, F. C. 574 (1898).

HARSHBERGER, J. W. 1533 (1917).

HARTMANN, A. $s$. ANGERER, K. v.

HaRTwich, C. u. Simon, N. 762 (1903).

HARVEY, W. W. 536 (1897).

HARZ, C. O. 270 (1885).

HASElhoff, F. U. MACH, F. 913 (1906).

HASHITANI, Y. 2059 (1927).

HASLAM, TH. P. 1114 (1910).

Hatano, J. 1823 (1924), 1904, 1905, 1906 (1925).

HATTORI, H. 612 (1899), 669 (1901).

HATTORI, Y. 1824 (1924), 1907 (1925).

HAUMANN, L. 704, 705 (1902).

HAURY, A. 613 (1899).

HÉE, A. 1978 (1926), 2070 (1927).

s. auch TERROINE, E. F.

HEIDER, A. 356 (1890).

HEIM, M. F. 420 (1893).

HEINRICHER, F. 499 (1896).

HeINSIUS, O. V. 670 (1901).

HEINZE, B. 76.3 (1903), 812 (1907), 870 (1905).

HENNEBERG, W. 1003(1908', 1979(1926).

HENNINGS, P. 457, 453 (1895), 641(1900), 764 (1903).

一, IINDAU, G., LINDNER P. u. NEGER, F. 871 (1905).

HENRY. S. NEES. V. ESENBECK, C. G.

HENRY, A. W. 1825 (1924).

HERING, G. 813 (1904).

HÉRISSEY, H. 500 (1896), 765 (1903), 2071 (1927).

u. LEBAS, C. 1168 (1911).

s. auch BOURQUELOT, É,

HERRENSCHWAND, F. V. 2072 (1927).

HERRICK, H. T. $s$. MAY. O. E.

HERTER, W. 1316 (1913), 1406 (1914), 1667 (1921).

— u. FORNET, A. 1534 (1917), 1589 (1919).

HERZOG, R. O.

— u. MELER, A. 1004 (1908), 1061 (1909).

- u. Polotzky, A. 1062 (1909).

- u. Ripke; O. 1005 (1908).

- u. SAladin, O. 1169 (1911). 
HeUSINGER. 18 (1821),

HeYes, T. F. u. Hor DEN, H. S. 1980 (1926).

HILDEBRANDT. 320 (1888).

HILGARD, T. 132 (1871).

HILL, A, C. 671 (1901).

HILLER, M. F. 706 (1902).

HIITNER, L. 707 (1902).

Hirsch, P. 2374 (1918).

HOCHE, CI. H. 872 (1905).

HOCHHEIM, K. 708 (1902).

Hodara, M. U. BeHDJEL, H. 1826(1924), 2413 (1925).

HoFFMANN. 149 (1874).

HOFFMANN, H. 85 (1860).

HÖHNEL, FR. V. 709 (1902), 1758 (1923).

HOLDEFLEISS. 250 (1883), 575 (1898).

HoldEN, G. W. 1464 (1915).

HoldeN, H. S. S. HEYES, T. F.

HOLDERER, M. 1063 (1909), 1115 (1910). s. auch BERTRAND, G.

Hollande, A. Ch. u. BeaUverie, J. 1496 (1916).

Hor,zER, H. S. JANKE, A.

HOOVER, F. P. 1235 (1912).

HOPFFE, A. 1590, 1591 (1919).

HOPPERT, C. 2403 (1924).

HöPPI, R, R. 1710 (1922).

HORI, S. 1115 (1910).

HorowiTZ-WIAASSOWA, I. M. 2198(1928).

HorTon, E. 1668 (1921).

Horvath, A. A. u. ShIN-HAO, L. 2073 (1927).

Hoshi, A. 1760 (1923). s. auch Oshima, $\mathrm{K}$.

HösTERMANN, G. u. NoAk, M. 1759 (1923).

HUGOUNENQ, I, u. FloRENCE, G. 1622, $2387(1920)$.

HUNGER. F. W. F. D. 614 (1899).

HuRsh, C. R. 2199 (1928).

Huss, H. 1407 (1914).

IKEDA, T. 1908 (1925).

INOUE, B. 1592 (1919).

INUI, T. 672, 673 (1901).

IRWIN, J. W. 1006 (1908).

ISAKOWITZ. 1054 (1909).

ISHIDA, A. $s$. YAMADA, S.

ISSATSCHENKO, B. L. 1007 (1908).
ITAYA, S. s. OshIma, K.

ITo, H. 1317 (1913).

ITo, M. 2074, 2075 (1927), 2200, 2201, 2202

(1928).

s. auch MrYake, K.

IWANOFF, I. 766 (1903).

IWANOFF (IWANOW), N. N. 1909, 1910

(1925), 2076 (1927), 2203 (1928).

IWANOFF, S. 710 (1902), 814 (1904).

IWASAKI, T. 1981 (1926).

IWATSURU, R. 1982 (1926).

JACOBSEN, K. A. S. EHRLICH, F.

JACoby, M. u. ShimizU, T. 1711 (1922).

JACQUOT, R. S. TERROINE, F. F.

JACZEWSKI, A. DE. 439 (1897), 501(1896). JAEGER. 13 (1816).

JAGER, I. DE. 1170 (1911).

JALOUSTRE, L. 1008 (1908).

JANKE, A.

— u. IHorzer, H. 2204 (1928).

— u. ZIKES: H. 2205 (1928).

JASEVOLI, G. 1761 (1923).

JAVILIIER, M. 615 (1899), 958, 959 (1907), 1009,1010 (1908), 1236, 1237, 1238 (19.12)

1318, 1319, 2369 (1913), 1408, 1409, 1410 1411, 1412 (1914).

- u. SAUTON, B. 1171 (1911).

u. TCHERNOROUTZKY, H. 1320, 1321 (1913).

s. auch BERTRAND, G.

JEGOROFE (JEGOROV), M. A. 1239 (1912). JELIIFFE, S. E. 537 (1897).

JENSEN, C. N. 1240 (1912).

JENTSCH, A. B. 1623 (1920).

JOACHIMOWITZ, M. 1535 (1917).

JOBL,ING, J. W. u. ARNOL,D, I. 1762 (1923).

Jochems, S. C. J. 1983 (1926).

JOFFE, J. S. s. WAKSMAN, S. A.

JOHAN-OL,SEN, S. O. 271(1885), 285, 286 (1886), 1241 (1912).

Johnson, A. H. 767 (1903).

JoHnson, J. W. 1763 (1923).

JoNG, D. A. DE. 1242 (1912), 1413 (1914).

JÖRGENSEN, A. 287 (1886), 357 (1890), 421

(1893), 2354 (1909).

JöRSTAD, I. 2206 (1928).

JoSEPHSON, K. 1827 (1924).

s. auch EULER, H. v.

JourdE, A.1011(1908). s.auch SARTORY, A. 
JoUsSFT, P. 768 (1903), 815 (1904).

JowETT, W. 1322 (1913).

JUHLER, J. J. 459, 460 (1895).

JUNITZKr, N. 960 (1907).

Kabashima, SH. 1911 (1925).

KавÁt u. Bubák. 87.3 (1905), 1012 (1908).

KaKEGAWA, T. 5. KaTO, M.

KAMBAYASHI, Y. 2207 (1928).

KANE, K. 1828 (1924), 1912 (1925).

KANITZ, A. 759 (1903), 2372 (1915).

KANTER, R. M. 770 (1903).

KAPUR, S. N. s. FALCK, R.

KARSTEN, P. A. 139 (1873).

KÁS̆, V. s. N亡̌MEC, A.

KaShiwaGi, K. 1669 (1921).

KaTO, M. u. KAKEGAWA, T. 1913 (1925).

KAto, S. 2077 (1927).

KATZ, J. 576 (1898).

KaUfFMaNN, G, s. Feiton, L. D. KaUfManN, M. 233, 234 (1882).

Kavina, K. 1559 (1918).

KAWAKITA. 577 (1898).

KaWAMOTO, T. 2208 (1928).

KAYSER, B. 771 (1903).

KAYSER, E. 578 (1898).

KEISSI,ER, K. VON. 1560 (1918).

KELLER, PH. 2209 (1928).

KELLERMAN, K. F. 772 (1903).

— u. MCBETh, J. G. 1243 (1912).

KELLNER, O. 451 (1895).

—, MORI, Y. u. NAGAOKA, M. 321

(1888), 358 (1890), 2319 (1893).

u NagaOKa, M. 422 (1893).

- NaGaOka, M. u. Kurashima, Y. 333 (1889).

KERR, I.. 874 (1905).

KICKX, J. 48 (1849), 109 (1867).

KIESEL, A. 1244 (1912), 1323, 1324 (1913), 1414 (1914).

KILLIAN, $\mathrm{CH}_{\text {H. }} 1764$ (1923).

Kinoshita, A. 1765 (1923).

Kinoshita, K. 2079 (1927).

KISCH, B. 1245 (1912), 1593 (1919).

KITA, G. 1246 (1912), 1325, 1326, 1327

(1913), 1415 (1914), 1535, 1537 (1917), 1561 (1918).

— u. Suzuki, K. 1766 (1923).

KiTANO, T. 2415 (1926).
Kitasato, T. 2079 (1927).

KIZEL, A. 1465 (1915).

KLEBERGER, K. 1624 (1920).

KLEBS, G. 2320 (1893), 502 (1896), 579

(1898), 642 (1900), 2210 (1928).

KLEIN, G., EIGNER, A. u, MÜLIER, H. 1984 (1926).

Klein, L. $s$. Challenger, F.

KLISSINNIS, N. 2404 (1924).

KI,ÖCKER, A. 1497 (1916), 1538 (1917), 1830 (1924).

— u. SchiöNNING, H. 462 1895), 503, 504 (1896).

KLOTZ, L. J. 1767 (1923).

KNAPP, H. 2080 (1927).

KNIEP. H. 2211 (1928).

KNISCHEWSKY, O. 961 (1907).

KNUDSON, L. 1328, 1329 (1913).

$\mathrm{K}_{N Y}$, L. 207 (1880), 218 (1881).

KoAna, F. 1985 (1926)

Kobayashi, T. s. Nakazawa, R.

$\mathrm{KOCH}, \mathrm{A}$.

- u. OELSNER, A. 1498 (1916).

- u. SEYDEL, S. 1247 (1912).

KосH, R. 219 (1881).

KOCH, W. u. REED, H. S. 952 (1907).

KOEHI,ER, B. 2081 (1927).

KOERNICKE, M. 816 (1907).

KoGA, T. 1768 (1923).

KoHL, F. G. 1013 (1908).

KÖHLER, P. 963 (1907).

KoHN, G. s. PRINGsheim, H.

KoHN, E. 423 (1893), 914 (1906).

- u. CZAPEK,F. 915 (1906).

KORACZEK, E. 76 (1857).

KoLKWITZ, R. 616 (1899), 1466 (1915).

Kominami, K. 1065 (1909).

KoNDO, K. 393 (1892).

KöNIG, J., SPIECKERMANN, A. u. BREMER, W. 674 (1901).

Koning, C. J. s. OUdemans, C. A. J. A. KOPACZEWSKI, W. 1330 (1913).

KOPELOFF, L. S. KOPEIOFF, N.

KOPEI,OFF, N. 1625 (1920). u. Byal, S. 1626 (1920).

u. KOPELOFF, L. 1594, 1595, 1596

(1919), 1627 (1920).

—, KOPELOFF, L. U. WELCOME, C. J. 1628 (1920).

Kopp, G. s. TERROINE, E. F.

KORDE, H. 1769 (1923). 
KORSCHEIT, O. 167 (1876), 187 (1878).

KOSCËLECKIJ, A. K. 1066 (1909).

KoshizawA, U. 1248 (1912).

KosInski, I. 711 (1902).

Koske, F. 875 (1905).

Kossowicz, A. 1249, 1250, 1251, 1252, 1253 (1912), 1331, 1332, 1333, 1334 (1913), 1416,1417, 1418, 1419 (1914), 1467 (1915), 1670 (1921).

— u. GRÖLLER, L. v. 1335 (1913).

- u. LOEW, W. 1336, 1337, 1338(1913).

KostyTsCHEW, S. 675 (1901), 712 (1902), 817,818 (1904), 965, 966 (1907), 1629 (1920), 2405 (1924), 2082 (1927).

—u. Afanassjewa, M. 1671 (1921), 1770 (1923).

- u. Filiasberg, P. 1630 (1920).

— u. TSCHESNOKOW, W. 2083(1927).

- u. TSWETKOWA, E. 1631 (1920), 1931 (1924).

KOTLJAR, E. 440 (1894).

KoZAI, Y. 643 (1900).

— u. I,OEW, O. 819 (1904).

—. YABE, K. 463 (1895).

KRAINSKIJ, A. V. 1254 (1912).

KRAMER, G. 1255 (1912).

KRAMER, O. 2406 (1924).

KRASNOSSEI,SKY, T. 820 (1904).

KRAUSE, C. S. NÖLLER. W.

KREMER, J. 505 (1896).

KREMER, W. $s$. STORM VAN LEEUWEN, W.

Krosby, P. 1986 (1926).

KRUSE, W. 2358 (1910).

Kubota, K. s. Matsumoto, $\mathrm{K}$.

KUDO, S. 1172 (1911).

KÜHL, H. 967 (1907), 1067, 1068, 1069 (1909), 1117 (1910), 1173 (1911), 1.339 (1913).

KUнN, R. 1771 (1923), 1832 (1924), 191 (1925).

u. GRUNDHERR, G. E. VON. 1984 (1926).

— u. MÜNCH, H. 2084 (1927).

u. WAGNER-JAUREGG, T. 1988 (1926).

KULLBERG; S. S. EULER, H. V.

KUMAGAWA, H. S. WILLSTÄTTER, R.

KUMANOMIDOH, S. 2212 (1928).

KUNSTMANN, H. 464 (1895).

Kurashima, Y. s. KFILNER, O.
KuRono, K. 1833 (1924), 2420 (1927).

- u. FujITA, E. 1989 (1926).

s. auch NEUBERG, C.

KURZWELLY, W. 773 (1903).

KÜSTER, E. 1014 (1908).

KYLIN, H. 1420 (1914).

LABORDE, V. 465 (1895), 538 (189\%).

LAFAR, F. 506 (1896), 580 (1898), 821 (1907).

LAGERHEIM, G. V. 774 (1903).

LANDRIEU, M. 2363 (1912).

LANG, F. J. u. GRUBAUER, F. 1772(1923).

I.ANGE, F. $s$. EHRLICH, F.

I.ANGERON, M. 1712, 1713 (1922), 1834 (1924), 2213 (1928).

LAPHAM, M. E. 2085 (1927).

LAPPALEINEN, H. 1256 (1912), 1597(1919).

LARA, N. A, 2214 (1928).

LASSALlie, H. u. Detas, R. 1990 (1926).

I,ATHAM, M. E. 876 (1905), 1070 (1909).

LAURENT, E. 331 (1889).

LAVES, W. S. HAHN, A.

I/AVIER, G. u. BIDOT, CH. 3394 (1922).

LAYCOCK, T. 2215 (1928).

LEBAS, C. $S$. HÉRISSEY, H.

LEBER, TH. 235 (1882).

I,EBERLE, H. S. BOAS, F.

LECLERG, F. L. u. SMITH, F. B. 2216 (1928).

LEE, H, A., WrILIER, D. M. U. BARNUM, C. C. 1991 (1926).

LEFÈVRE, E. S. THOM, СH.

LEIBOWITZ, J.

u. Mechlinski, P. 1992, 1993

(1926)

s. auch NEUBERG, C.

LEIDY, J. 158 (1875).

LENDNER, A. 539 (1897), 775 (1903).

LENORMAND, C. $s$. BODIN, F.

I EO. 507 (1896).

I.EPIERRE, CH. 508 (1896), 1257 (1912), 1340, 1341, 1342, 1343, 1344, 1345 (1913), 1421 (1914).

LESAGE, P. 713 (1902), 822 (1904).

LESZCZYNSKI, R. v. u. EPLER, R. 1994 (1926).

I.EUNIS, J. u. FRANK, A. B. 2311 (1886).

LÉVEILLÉ, J. H. 57 (1851).

LEVI, M. $s$. GALEOTTI, C. 
LEEWIS, P. M. 2086 (1927).

IICHTENSTEIN, S. $S$. PRINGSHEIM, H.

I.ICHTHEIM, L. 236, 237 (1882).

LIEBSCHER, 220 (1881).

LILIY, C. H. 1915 (1925).

IIND, J. 1346 (1913).

L/ND, K. 581 (1898).

IINDAU, G. 823, 824 (1904), 968 (1907), 2364 (1912).

s. auch WINTER, G. u. HENNINGS, P.

LINDEGREN, C. C. S. WALKER, J. C.

LINDET, I. 1422 (1914).

IINDNER, J. 1423 (1914).

IIINDNER, K. 1347 (1913).

IINDNER, P. 2344 (1903), 1015, 1016(1908), 2355 (1909), 1258, 1259 (1912).

— u. CZISER, S. 1260 (1912).

- u. MATTHES, P. 825 (1904).

s. auch H.ENNINGS, P.

IIIND', W. 288 (1886), 335 (1889).

LiNhardT, K. $s$. NeUbERG, C.

$\mathrm{I}_{\mathrm{I}} \mathrm{INK}$, H. F. 11 (1809), 14 (1816), 23(1824), 28 (1829).

LINNÁ, C. DE. 3 (1753).

IINOSSIER, G. 370, 371, 372 (1891), 1118 (1910).

u. Roux, G. 359 (1890).

IIPMAN, СH. B. 1174 (1911).

— u. BURGESS, P. S. 1468 (1915).

LITTAUER, F. S. REICHER'T, I.
LivingsTON, B. E. 2345 (1903).

LOBIK, A. J. 1995 (1926).

I,ODE, A. 714 (1902).

LOEPER, M. S. BRAULT, A.

ILOEW, O. 776, 777 (1903), 1916 (1925).

s. auch KozAI, Y.

LoEw, W. s. Kossowicz, A.

LÖFFLER, H. 1835 (1924).

LöHNIS, F. 1119 (1910).

LOLANIER, M. 289 (1886).

LOMBARDO, F. 1996 (1926).

L.OPEZ, J.-LOMBA. 1773 (1923), 1836(1924).

Loudon, J. C. 68 (1855).

LOWE, J. 77 (1857), 79 (1858).

I_öwschin, A. 1017 (1908).

I UUBS, H. A. s. ClaRk, W. M.

LUCET. S. COSTANTIN.

LUCET, A. 509 (1896), 617 (1899).

L UCKSCH, F. 715 (1902).

LÜDERITZ, C. 360 (1890).

I,UDWIG, F. 394 (1892).

I,UDWIG, O. 2217 (1928).

L.UTZ, L. 582 (1898), 644. (1900), 716, 717 (1902), 877, 878, 879, 880 (1905), 969 (1907).

LUTZ, O. 1071 (1909).

I.wow, S. 1175 (1911).

I/YNCH, K. M. 1774 (1923).

(Schluss folgt.)

\section{おにゆりノ染色體二就テ}

\section{竹中要、永松土巳}

Y. TAKENAKA and T. NAGamaTsu: On the Chromosomes of Lilinm tigrinum, KER-GAWL.

おにゆりノ花粉母細胞ノ細胞學的研究入 19 世紀ノ未葉ヨリ 20 世紀ノ始メニカ ケテ|Coulter, Chamberlain, Schaffner 等ニョツテナサレ、其つ染色體八單 數ニテ 12 倜ナルコト知ラレ、CHAMBERLAIN 八其ノ花粉管內ノ不規则ナル核分裂 ニョル多數ノ核ヨ觀察シ、其レト原枼體トヨ關聯シテ說明セリ。余等八おにゆりノ 不稔性ナル珠芽ヨ生ズルトハ恐ラク何等カノ核學的根據ヨ有スルモノナルベシト推 测シ、イササカ研究スル所アリタレバ以下ンレフ述ベン。 